\section{Arrestin psychosis}

\section{By Tim Fulmer, Senior Writer}

Researchers from Duke University and The University of North Carolina at Chapel Hill have used the antipsychotic drug Abilify aripiprazole as a scaffold to design a new class of antipsychotic compounds that showed fewer side effects in mice. ${ }^{1}$ The researchers are currently negotiating a licensing deal for the compounds with an undisclosed pharma.

Antipsychotics typically exert their effects by either agonizing or antagonizing the dopamine D2 receptor on neurons, thus altering dopaminergic signaling in the brain and reducing symptoms in diseases such as schizophrenia, depression and bipolar disorder.

However, long-term use of antipsychotics is associated with serious motor, cardiovascular and metabolic side effects. Those side effects lead to discontinuation of treatment in as many as one-third of schizophrenia patients. $^{2}$

The reason for the side effects may lie with the D2 receptor itself, which activates multiple downstream signaling pathways when it binds to the drugs. ${ }^{3}$ Because some downstream pathways may contribute to efficacy while others trigger side effects, the net result is a suboptimal therapeutic index.

A Duke-North Carolina team led by Bryan Roth, Jian Jin and Marc Caron decided to try to design a D2 receptor ligand that would activate antipsychotic pathways without increasing the activity of pathways associated with side effects.
Roth is a professor of pharmacology at UNC's School of Medicine. Jin is an associate professor and director of medicinal chemistry at the Center for Integrative Chemical Biology and Drug Discovery at UNC. Marc Caron is a professor of cell biology at Duke and an investigator at the Howard Hughes Medical Institute.

The team was guided by previous work in multiple labs that showed G protein-coupled receptors (GPCRs) such as the D2 receptor signal not only through well-known $G$ protein pathways but also through the $\beta$-arrestin pathway (see Figure 1, "Putting a bias into antipsychotics"). In rodent models of psychiatric disorders, the $\beta$-arrestin pathway played a key role in mediating behavioral responses to some dopaminergic drugs. ${ }^{4-6}$

Thus, they set out to design a D2 receptor ligand that was biased toward activating the $\beta$-arrestin pathway over $G$ protein pathways potentially responsible for side effects.

For a scaffold, the researchers chose Abilify because it is the only marketed D2 receptor agonist. All the other marketed drugs that target the receptor, including first- and second-generation antipsychotics like the generics clozapine and risperidone, are antagonists of the D2 receptor and perhaps not as suitable as scaffolds for designing a $\beta$-arrestin-biased agonist.

Abilify, a partial agonist of the D2 receptor, is marketed by Otsuka Pharmaceutical Co. Ltd. and Bristol-Myers Squibb Co. to treat neurological diseases including bipolar disorder, mania, depression and schizophrenia.

The team tested 150 Abilify derivatives in vitro and found 3-UNC9975, UNC0006 and UNC9994-that bound the D2 receptor and agonized $\beta$-arrestin signaling without activating other downstream pathways mediated by $\mathrm{G}$ proteins.

UNC9975 showed the best CNS penetration in mouse pharmacokinetic studies and thus became the main focus of subsequent experiments. In two mouse models of chemically induced

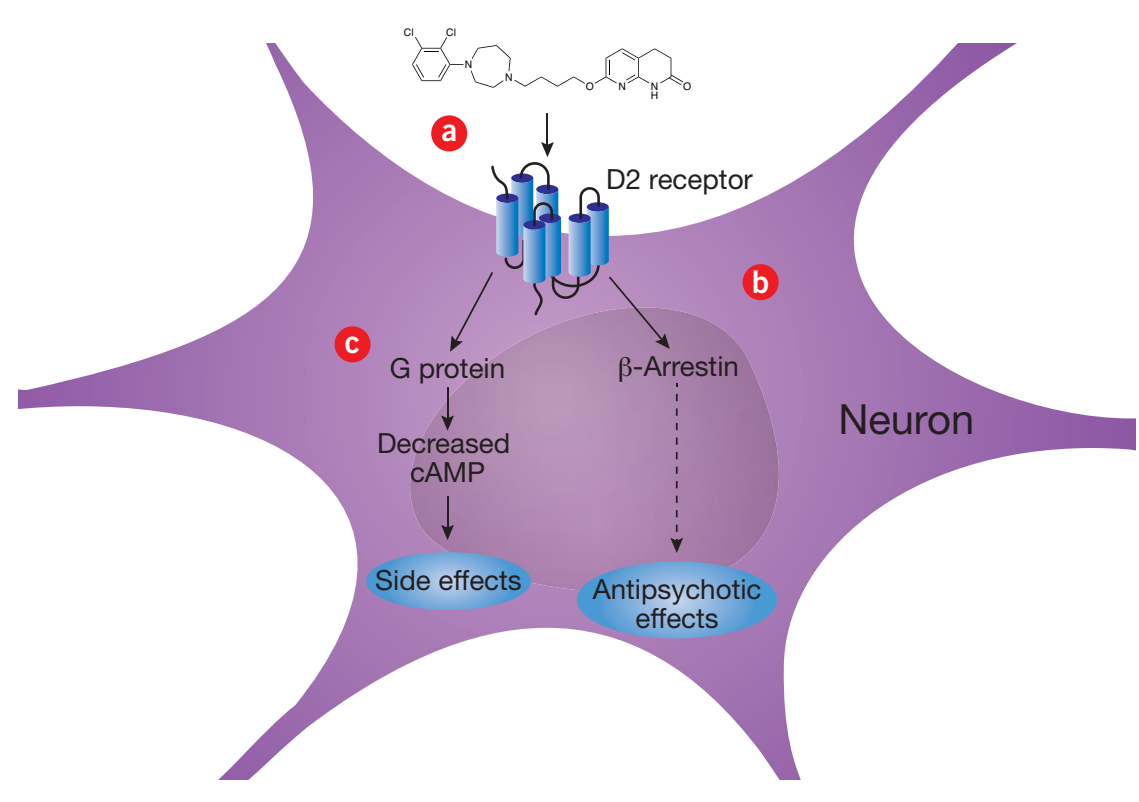

Figure 1. Putting a bias into antipsychotics. A team from The University of North Carolina at Chapel Hill and Duke University has designed a class of antipsychotic compounds called $\beta$-arrestin-biased ligands that could have a better therapeutic index than existing drugs to treat schizophrenia and other neuropsychiatric disorders.

Using the antipsychotic drug Abilify as a synthetic scaffold, the researchers designed a biased agonist that bound the dopamine D2 receptor [a] and activated downstream signaling through the $\beta$-arrestin pathway [b] without agonizing G protein-mediated pathways [c]. In mice, the compound showed antipsychotic effects without triggering neurological side effects.

Abilify aripiprazole is marketed by BristolMyers Squibb Co. and Otsuka Pharmaceutical Co. Ltd. to treat depression, bipolar disorder and schizophrenia. 
psychotic behavior, UNC9975 decreased hyperlocomotion compared with vehicle.

In a third mouse model of drug-induced catalepsy, which serves as a proxy for some of the neurological side effects of antipsychotics, UNC9975 did not induce catalepsy compared with the generic antipsychotic haloperidol.

Data were published in the Proceedings of the National Academy of Sciences.

"Just as Abilify has generally milder side effects than earlier antipsychotics like haloperidol and risperidone, we think our $\beta$-arrestinbiased agonists could be yet milder than Abilify, thus continuing the trend of antipsychotic compounds with an improved therapeutic index," corresponding author Jin told SciBX.

The compounds described in the paper "could point the way toward a new class of antipsychotic drugs," said Robert Lefkowitz, a professor

"Just as Abilify has generally milder side effects than earlier antipsychotics like haloperidol and risperidone, we think our $\beta$-arrestin-biased agonists could be yet milder than Abilify, thus continuing the trend of antipsychotic compounds with an improved therapeutic index."

- Jian Jin,

The University of North Carolina at Chapel Hill of medicine, pathology and biochemistry at Duke who was not involved in the study. "Though the compounds require further characterization and optimization, it seems the researchers have found a way to selectively agonize the $\beta$-arrestin pathway without activating $G$ protein-mediated pathways that could contribute to toxicity."

In 1990, Lefkowitz first described the role the $\beta$-arrestin pathway plays as a regulator and signaling mediator of GPCRs. He also is a cofounder of Trevena Inc., which is focused on developing GPCR ligands that are biased to selectively activate or block pathways that play a role in cardiovascular, CNS and inflammatory diseases.

Trevena's lead compound is TRV120027, a $\beta$-arrestin-biased ligand of the angiotensin II type 1 receptor (AGTR1). The compound is in Phase II testing to treat acute heart failure.

\section{Finding the right disease}

The next step for the North Carolina team is to identify the diseases for which the $\beta$-arrestin-biased agonists are best suited.

One approach would be to test the compounds "in a broad range of behavioral and disease models, noting how the biased ligands differ beneficially from reference compounds like aripiprazole," said Jonathan Violin, cofounder and head of biology at Trevena.
Another option would be "to compare the $\beta$-arrestin-biased agonists against one another in a panel of rodent models to get a better idea of which compounds are better suited for treating positive or negative symptoms of schizophrenia," said Jean-Martin Beaulieu, assistant professor of psychiatry and neuroscience at Laval University. "There are many genetic models of schizophrenia that might allow the researchers to study the effects of their compounds on key aspects of disease such as cognitive deficits and reduced social interaction."

In 2008, Beaulieu, Lefkowitz and Caron published in Cell that $\beta$-arrestin signaling mediated the effects of the mood stabilizer lithium on mouse behavior. ${ }^{7}$

Jin said he, Roth and Caron will continue to collaborate "to further optimize the $\beta$-arrestin-biased agonists, testing them first in in vitro functional assays, then subsequently in behavioral and genetic models of neuropsychiatric disorders. In those animal models, we will also focus on the effects of chronic treatment."

UNC holds issued patents on the compounds. According to Jin, the team is in negotiations with an undisclosed pharma that is considering licensing the compounds from UNC, but he declined to disclose additional details.

Fulmer, T. SciBX 4(44); doi:10.1038/scibx.2011.1224

Published online Nov. 10, 2011

\section{REFERENCES}

1. Allen, J.A. et al. Proc. Natl. Acad. Sci. USA; published online Oct. 24, 2011; doi:10.1073/pnas.1104807108

Contact: Jian Jin, The University of North Carolina at Chapel Hill, Chapel Hill, N.C.

e-mail: jianjin@email.unc.edu

Contact: Bryan L. Roth, same affiliation as above

e-mail: bryan_roth@med.unc.edu

2. Nosè, M. et al. Trials 10, 31 (2009)

3. Beaulieu, J.-M. et al. Annu. Rev. Pharmacol. Toxicol. 49, 327-347 (2009)

4. Beaulieu, J.-M. et al. Cell 122, 261-273 (2005)

5. Rajagopal, S. et al. Nat. Rev. Drug Discov. 9, 373-386 (2010)

6. Del'Guidice, T. et al. Front. Neuroanat.; published online Sept. 6, 2011; doi:10.3389/fnana.2011.00058

7. Beaulieu, J.-M. et al. Cell 132, 125-136 (2008)

\section{COMPANIES AND INSTITUTIONS MENTIONED}

Bristol-Myers Squibb Co. (NYSE:BMY), New York, N.Y. Duke University, Durham, N.C.

Howard Hughes Medical Institute, Chevy Chase, Md. Laval University, Beauport, Quebec, Canada Otsuka Pharmaceutical Co. Ltd., Tokyo, Japan Trevena Inc., King of Prussia, Pa.

The University of North Carolina at Chapel Hill, Chapel Hill, N.C. 